\title{
Predictors for Pediatric Blunt Cerebrovascular Injury (BCVI): An International Multicenter Analysis
}

\author{
Christian D. Weber ${ }^{1}$ (1) $\cdot$ Rolf Lefering $^{2} \cdot$ Matthias S. Weber $^{3} \cdot$ Georg Bier $^{4}$. \\ Matthias Knobe $^{1} \cdot$ Miguel Pishnamaz $^{1} \cdot$ Philipp Kobbe $^{1} \cdot$ Frank Hildebrand $^{1}$. \\ TraumaRegister DGU
}

Published online: 3 June 2019

(C) Société Internationale de Chirurgie 2019

\begin{abstract}
Introduction Practice guidelines for adult BCVI patients have been implemented recently, but data for this devastating injury pattern in children are still limited. An international multicenter analysis was performed to characterize BCVI in the pediatric population.

Methods The TraumaRegister DGU ${ }^{\circledR}$, a prospectively maintained database, was analyzed (01/2002-12/2015). Pediatric patients (0-17 years) with major injuries [Injury Severity Score (ISS) $\geq 9$ points] were included. BCVI was divided into carotid artery injury and vertebral artery injury (VAI). Data of demographics, injury, imaging, therapy, and outcome characteristics were analyzed with SPSS (Version 25, IBM Inc., Armonk, NY).

Results The study cohort included 8128 pediatric trauma patients. We identified 48 BCVIs in 42 children, resulting in an overall prevalence of $0.5 \%$. Carotid injuries were diagnosed more frequently $(n=30 ; 0.4 \%)$ when compared to VAIs $(n=12 ; 0.1 \%)$. The coincidence of head $(p=0.028)$, facial $(p \leq 0.001)$, chest $(p \leq 0.001)$, and spinal injuries $(p \leq 0.001)$ was higher in BCVI patients. The risk for thromboembolic complications $(8.3 \%$ vs. $1 \%, p=0.026)$ and in-hospital mortality $(38.1 \%$ vs. $7.7 \%, p \leq 0.001)$ was excessive in children with BCVI. We identified various predictors for pediatric BCVI and quantified the cumulative impact of these risk factors.

Conclusion BCVI is more uncommon in pediatric than in adult trauma patients. Due to the considerable relevance of this injury for both children and adults, special attention should be paid to this entity and associated complications in the early treatment phase after severe pediatric trauma, especially in high-risk children.
\end{abstract}

Christian D. Weber

chrweber@ukaachen.de

1 Department of Orthopedics and Trauma Surgery, RWTH Aachen University Medical Center, Pauwels Street 30, 52074 Aachen, Germany

2 Institute for Research in Operative Medicine (IFOM), Witten/ Herdecke University, Cologne, Germany

3 Department of Pediatric Intensive Care Medicine, RWTH Aachen University, Aachen, Germany

4 Department of Diagnostic and Interventional Neuroradiology, Tübingen University Hospital, Tübingen, Germany

5 Committee on Emergency Medicine, Intensive Care and Trauma Management (Sektion NIS) of the German Trauma Society (DGU), Berlin, Germany

$\begin{array}{ll}\text { Abbreviations } \\ \text { ACCP } & \text { American College of Chest Physicians } \\ \text { AIS } & \text { Abbreviated Injury Scale } \\ \text { BCVI } & \text { Blunt cerebrovascular injury } \\ \text { BSF } & \text { Basilar skull fracture } \\ \text { CAI } & \text { Carotid artery injury } \\ \text { CI } & \text { Confidence interval } \\ \text { CTA } & \text { Computed tomography angiogram } \\ \text { DGU } & \text { Deutsche Gesellschaft für Unfallchirurgie/ } \\ & \text { German Trauma Society } \\ \text { ER } & \text { Emergency room } \\ \text { GCS } & \text { Glasgow Coma Scale } \\ \text { ICU } & \text { Intensive Care Unit } \\ \text { ISS } & \text { Injury Severity Score }\end{array}$




$\begin{array}{ll}\text { LOS } & \text { Length of stay } \\ \text { MCA } & \text { Motorcycle accident } \\ \text { mmHg } & \text { Unit millimeters of mercury } \\ \text { MOF } & \text { Multiple organ failure } \\ \text { MRA } & \text { Magnetic resonance angiography } \\ \text { MVC } & \text { Motor vehicle collision } \\ \text { OR } & \text { Odds ratio } \\ \text { SCCM } & \text { Society of Critical Care Medicine } \\ \text { SD } & \text { Standard deviation } \\ \text { SOFA } & \text { Sequential organ failure assessment score } \\ \text { TR-DGU } & \text { TraumaRegister DGU } \\ \text { VAI } & \text { Vertebral artery injury }\end{array}$

\section{Introduction}

In adult trauma patients, blunt cerebrovascular injuries (BCVIs) have been identified as a rare but devastating injury pattern affecting around $1-2.7 \%$ of multiply injured patients $[1,2]$. Furthermore, BCVIs were associated with a substantial risk for major complications and poor outcome $[3,4]$. Based on advanced imaging techniques, an increasing incidence of BCVI has been observed in adult patients. Also, distinct pharmacotherapeutic and interventional treatment algorithms have been implemented for the adult trauma population to avoid devastating neurologic sequelae. These algorithms also consider the specific aspects in cases of concomitant injuries of solid organs or the brain [5-7].

In contrast to adult patients, data on the relevance of BCVI and optimal diagnostic and therapeutic management in the pediatric trauma population remain sparse. However, children might present specific age-related factors, which can affect the characteristics of this entity in the pediatric trauma population. Among these, anatomic particularities, such as an increased head-body ratio, an immature neck musculature, and higher ligamentous laxity can result in increased forces on the cervical-thoracic junction in hyperextension or rotational injury. These aspects might increase the risk of BCVI. In contrast, a significantly lower incidence of arteriosclerotic changes of the cerebrovasculature may represent a potential protective factor [8].

Previous studies reported an incidence of pediatric BCVI between 0.095 and $0.9 \%$. However, all but one of these studies included only a very limited number of individuals [9]. The low incidence of BCVI reported in these studies might be related to an underreporting due to both the absence of current screening guidelines and the use of inadequate imaging techniques [10-15].

Focusing on potential risk factors for BCVI, similar predictors for the adult population were described [16].
Others found a trend toward an association with additional injuries (e.g., basilar skull fractures, intracranial hemorrhage) and the presence of BCVI [17].

Due to the high number of included patients, registry studies are a promising approach to increase knowledge about epidemiologic and BCVI-related characteristics. Therefore, we reviewed a large international trauma databank to elucidate the following questions:

1. What is the incidence of carotid and vertebral artery injury in pediatric trauma patients?

2. Do children feature BCVI specific risk factors?

3. Which major complications and outcomes are observed in pediatric BCVI (e.g., thromboembolic events, MOF, mortality)?

\section{Patients and methods}

\section{Inclusion and exclusion criteria}

All pediatric patients (aged 0-17 years) with severe injuries (ISS $\geq 9$ points) after admission to a participating trauma center in a German-speaking country (Germany, Austria, Switzerland) between January 2002 and December 2015 were included in this study (Fig. 1). Patients transferred out to another center within $48 \mathrm{~h}$ after admission were excluded due to missing outcome data and to exclude the chance of double counting $(n=700)$. However, all cases transferred in $(n=940)$ were included to prevent bias in prevalence rates.

\section{TraumaRegister DGU ${ }^{\circledR}$ and data acquisition}

The TraumaRegister DGU ${ }^{\circledR}$ of the German Trauma Society (Deutsche Gesellschaft für Unfallchirurgie, DGU) was founded in 1993 [18]. The aim of this multicenter database is pseudonymized and standardized documentation of severely injured patients. Data are collected prospectively in four consecutive time phases, from the site of the accident until discharge from hospital: (A) pre-hospital phase, (B) emergency room and initial surgery, (C) intensive care unit, and (D) discharge. The documentation includes detailed information on demographics, injury patterns, comorbidities, pre- and in-hospital management, a course on the intensive care unit, and relevant laboratory findings that include data on transfusions and outcomes for each individual. The inclusion criterion is admission via emergency room with subsequent ICU/ICM care, or admission to the hospital with vital signs and early death before admission to ICU. The infrastructure for documentation, data management, and data analysis is provided by the Academy for Trauma Surgery (AUC-Akademie der 
Fig. 1 Description of the study cohort recruitment with sample sizes
Total registry cohort

Germany/Austria/Switzerland

2002-2015

$n=212,068$

Adults excluded ( $>17$ years) $n=196,805$

Pediatric trauma patients

$n=15,263$

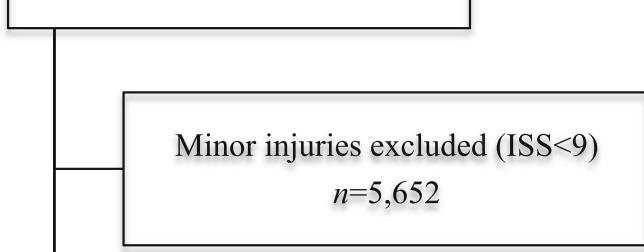

Penetrating $(n=331)$ or unknown $(n=425)$ mechanisms excluded

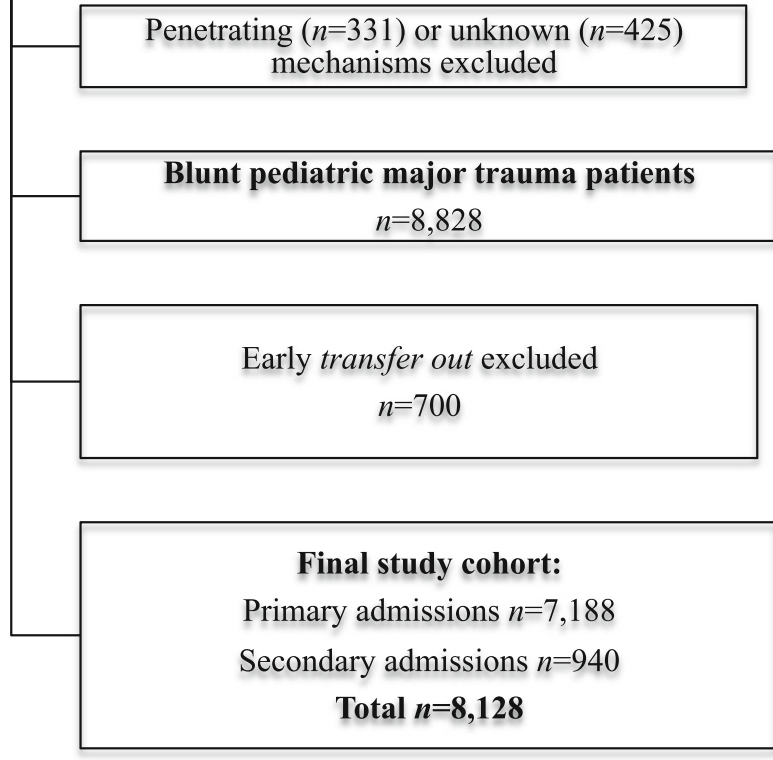

Unfallchirurgie $\mathrm{GmbH}$ ), a company affiliated with the German Trauma Society.

Scientific leadership is provided by the Committee on Emergency Medicine, Intensive Care and Trauma Management (Sektion NIS) of the German Trauma Society. The participating hospitals submit pseudonymized data into a central database via a web-based application. Scientific data analysis is approved according to a peer review procedure established by Sektion NIS.

The participating hospitals are primarily located in Germany (90\%), but a rising number of hospitals of other countries contribute data as well. (At the moment, Austria, Switzerland, Belgium, China, Finland, Luxemburg, Slovenia, The Netherlands, and the United Arab Emirates participate in this database.)

Currently, approximately 34,000 cases from more than 650 hospitals are entered into the database per year. Participation in TraumaRegister $\mathrm{DGU}^{\circledR}$ is voluntary. For hospitals associated with the TraumaNetzwerk DGU ${ }^{\circledR}$, 
however, the entry of at least a basic data set is obligatory for reasons of quality assurance. The present study is in line with the publication guidelines of the TraumaRegister DGU $^{\circledR}$ and registered as TR-DGU Project ID 2017-007.

\section{Definitions}

\section{Injury severity}

Since 2009, coding has followed a uniform protocol, and the data management has been previously described [18]. All injuries were coded according to the Abbreviated Injury Scale (AIS; Version 2005/Update 2008, Association for the Advancement of Automotive Medicine, Barrington, IL). The severity of injuries was documented as 1 (minor), 2 (moderate), 3 (severe, not life-threatening), 4 (serious, life-threatening), 5 (critical, survival uncertain), or 6 (maximum, currently untreatable) [19, 20].

\section{$B C V I$}

Identification according to AIS codes: carotid artery injury (CAI) codes: $3202 x x$ and 3204xx, and vertebral artery injury (VAI) codes: 3210xx. Computed tomography angiogram (CTA) was applied in primary BCVI screening in the majority of cases. CTA imaging performed after admission to the ICU or any other subsequent imaging studies (e.g., conventional angiography, MRA, and Doppler ultrasound) performed after the emergency phase for BCVI confirmation or re-evaluation were not documented. However, the trauma registry captures the definitive diagnosis (type/severity of BCVI) at the time of discharge or death. Modifiers of the main AIS code for injury severity allow differentiation between dissections (Biffl Grade II), transections (Biffl Grade V), occlusions (Biffl Grade IV), and bilateral injuries. While the AIS codes correspond to the Biffl grading system in some ways, the comprehensive Biffl classification (I-V) has not been implemented into the registry during the study period.

\section{Thromboembolic events}

The registry captures thromboembolic complications in the further hospital course. An acute post-traumatic infarction coded as AIS 140676.3 was considered as a stroke in this study. Stroke was diagnosed according to the current World Health Organization (WHO) definition [21].

This type of documentation was available in the standard documentation protocol, which is used in the majority of trauma centers that manage BCVI patients. No imputation or missing data treatment was performed. A mismatch analysis excluded cases with duplicate documentation.
Multiple organ failure (MOF)

Organ failure was defined as 3 or 4 points in the SOFA score [22]. MOF was present if two or more organ systems failed [23]. These data were available only in patients with standard documentation.

\section{Mortality}

Mortality was defined as in-hospital death from any cause.

\section{Statistical analysis}

Categorical data were presented as frequencies and percentages. Metric variables were reported as means and standard deviation (SD). In case of a skewed distribution, the median was also given. The Chi-square test was used for comparison of categorical variables, and the MannWhitney $U$ test was applied for metric variables. Bivariate logistic regression analyses were performed to elucidate the possible impact of various risk factors on the development of BCVI. Results are considered statistically significant if $p<0.05$. The analysis was performed with SPSS for Windows (Version 25, IBM Inc., NY, USA).

\section{Results}

During the study period, 8128 pediatric trauma patients fulfilled the inclusion criteria. Of these, a total of 42 children suffered from BCVI ( $0.5 \%$ overall incidence), and 8086 pediatric trauma patients were included as the control group. The detailed selection of the study patient population is described in Fig. 1.

\section{Blunt cerebrovascular injuries}

In the BCVI cohort, 30 children suffered from 35 carotid artery injuries, and 12 individuals sustained 13 vertebral artery injuries. There was no individual with combined CAI and VAI. The CAIs involved 20 dissections, 5 ruptures/pseudoaneurysm, and 5 bilateral injuries. VAIs included 5 dissections, 2 transections, 4 occlusions, and 1 bilateral injury (Fig. 2).

\section{Demographics}

Mean age and sex were comparable for both patient groups. The age distribution of affected children is presented in Fig. 3. The majority of patients were of male gender $(n=5441)$ in both groups (Table 1). BCVI patients presented with a significantly higher mean ISS when 


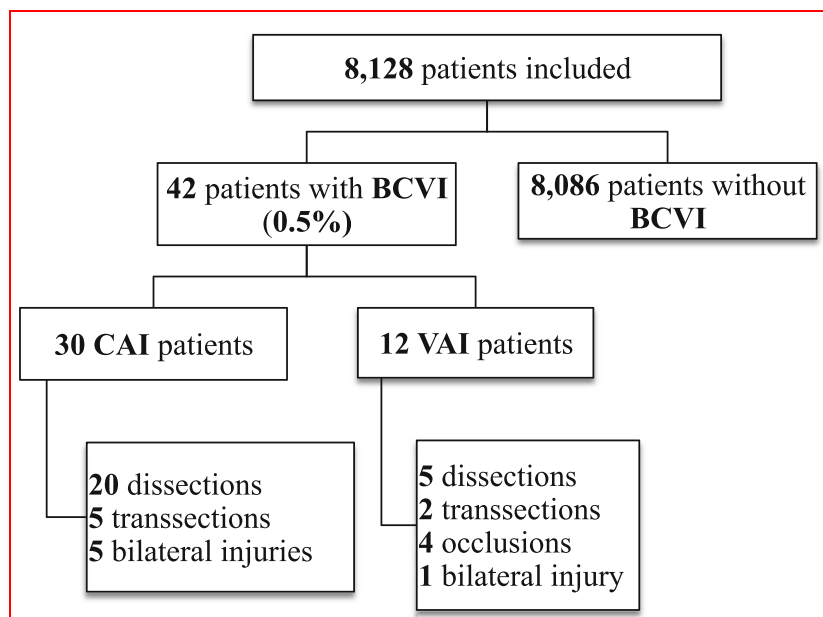

Fig. 2 Flow diagram of study population

compared to the control group (ISS 39 vs. 21 points; $p \leq 0.001$ ).

High-energy mechanisms and road traffic accidents were common underlying injury mechanisms (Table 1; in particular, motor vehicle crashes (MVC) were more frequently observed in the pediatric BCVI population $(85.4 \%$ vs. $70.4 \%, p=0.039)$. CAI patients had a mean age of 13.3 years and VAI patients had a mean age of 14.5 years. A separate breakdown of CAI- and VAI-associated mechanisms of injury is presented in Fig. 4.

\section{Concomitant injuries and early physiology}

BCVI patients more frequently presented with head injuries $(73.8 \%$ vs. $56.2 \% ; p=0.028)$ and basilar skull fractures $(31.0 \%$ vs. $16.6 \% ; p=0.020)$. Also, facial $(p \leq 0.001)$, chest $(p \leq 0.001)$, and spinal injuries ( $p$ $\leq 0.001$ ) were observed more frequently. A significant difference was found in the rate of cervical spine injuries ( $31.0 \%$ vs. $2.9 \% ; p \leq 0.001)$. Further details of the anatomic injury distribution are described in Table 2. Regarding physiologic parameters, a regular pupil reaction was less frequently documented in BCVI patients $(48.1 \%$ vs. $82.9 \%, p \leq 0.001$ ). Both a loss of consciousness (LOC; GCS $\leq 8$ points; $71.3 \%$ vs. $26.4 \%, p \leq 0.001)$ and a higher incidence of pre-hospital intubation $(83.3 \%$ vs. $38.8 \%, p \leq 0.001$ ) were more frequently reported in pediatric BCVI patients. The incidence of shock at the scene and at ER admission was increased in patients with $\mathrm{BCVI}$, resulting in a higher requirement for blood transfusions ( $45.7 \%$ vs. $10.6 \%, p \leq 0.001)$. A coagulopathy was more frequently observed in children with BCVI (74.7\%) when compared to the control group (29.6\%).

\section{Imaging, management, and complications}

Independently of the presence of BCVI, the majority of children in the control and BCVI groups underwent immediate head/neck computerized tomography (CT) ( $85.3 \%$ vs. $94.4 \%, p=0.16)$ or whole-body CT $(64.6 \%$ vs. $86.1 \%, p=0.008)$. Two BCVI cases did not undergo CT

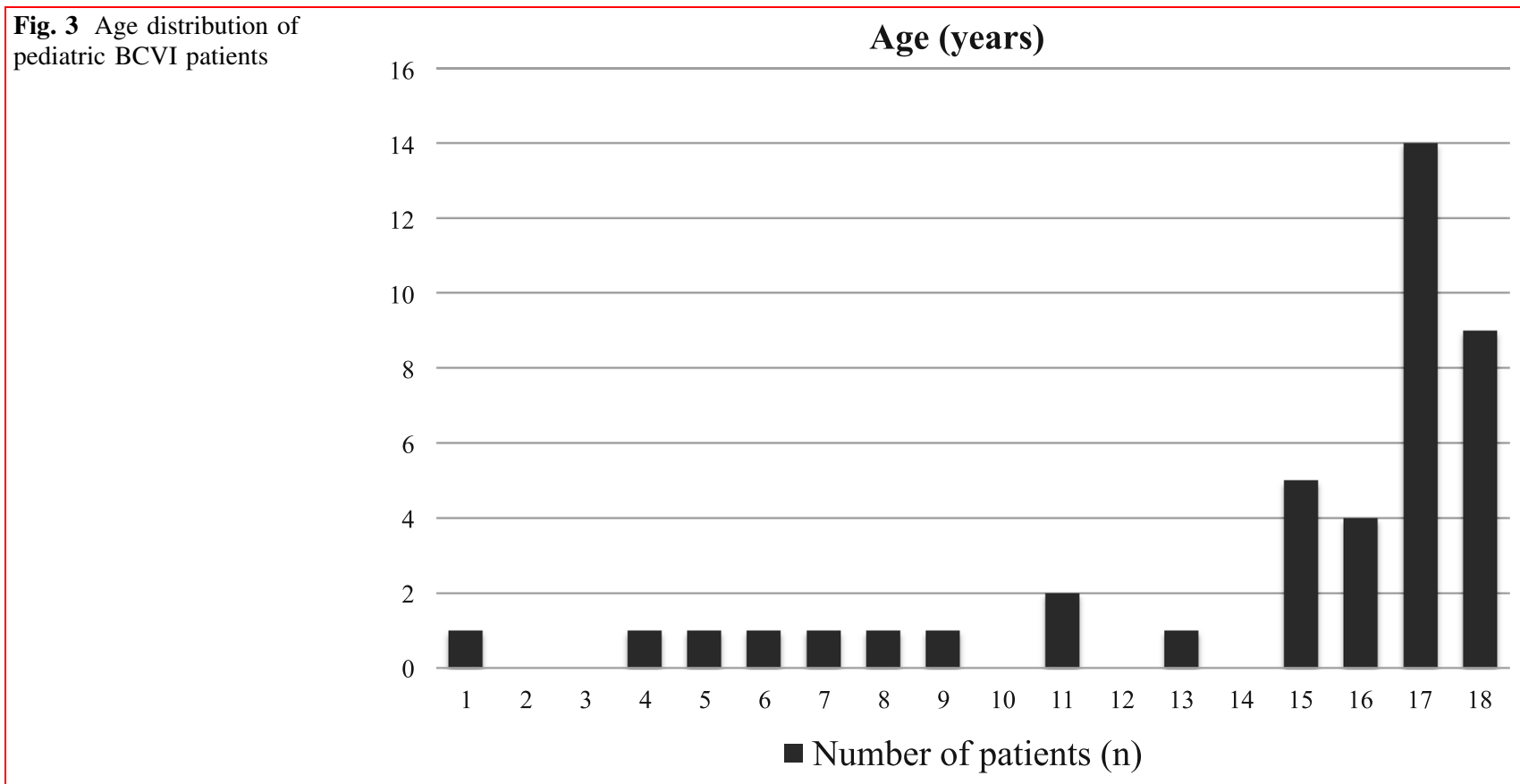


Table 1 Patient characteristics

\begin{tabular}{|c|c|c|c|}
\hline & Non-BCVI & $\mathrm{BCVI}$ & $p$ \\
\hline Male sex: $\%(n)$ & $67.1 \%(5414)$ & $64.3 \%(27)$ & 0.74 \\
\hline Age: mean (SD), years & $12(5)$ & $14(4)$ & 0.08 \\
\hline ISS: mean (SD), points & $21(12)$ & $39(15)$ & $\leq 0.001$ \\
\hline Motor vehicle crash (MVC) & $70.4 \%(5440)$ & $85.4 \%(35)$ & 0.039 \\
\hline Regular pupillary reaction, $\%(n)^{\mathrm{b}}$ & $82.9 \%(4315)$ & $48.1 \%(13)$ & $\leq 0.001$ \\
\hline Glasgow Coma Scale, $\leq 8$ points, $\%(n)$ & $26.4 \%(1764)$ & $71.4 \%(25)$ & $\leq 0.001$ \\
\hline Shock at scene $(\mathrm{SBP} \leq 90 \mathrm{mmHg}), \%(n)$ & $17.5 \%(1062)$ & $41.4 \%(12)$ & 0.002 \\
\hline Shock at ER admission, $\%(n)^{\mathrm{a}}$ & $12.1 \%(779)$ & $33.3 \%(11)$ & $\leq 0.001$ \\
\hline Coagulopathy at admission ${ }^{\mathrm{ab}}$ & $29.6 \%(2129)$ & $74.4 \%(29)$ & $\leq 0.001$ \\
\hline
\end{tabular}

ER emergency room, $S B P$ systolic blood pressure

${ }^{a}$ Only primary admitted cases $(\beta)$ available for cases with standard documentation

${ }^{\mathrm{b}}$ Definition: INR $>1.2$, Quick $<70$, Thrombocytes $<100,000$ or PTT $>40 \mathrm{~s}$

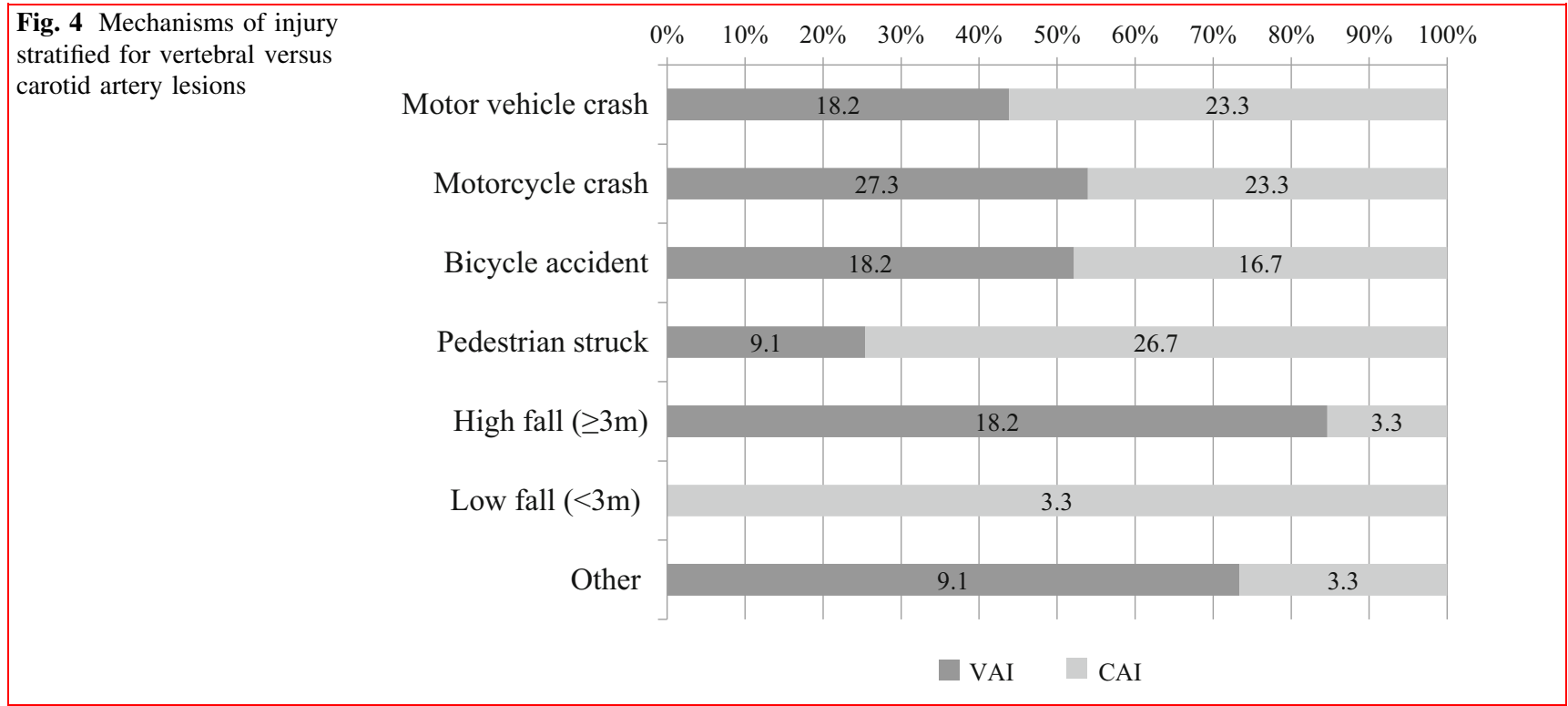

Table 2 Patterns of injury

\begin{tabular}{lllr}
\hline & Non-BCVI & BCVI & $p$ \\
\hline Head injury (AIS $\geq 2)$ & $56.2 \%(4542)$ & $73.8 \%(31)$ & 0.028 \\
Basilar skull fracture & $16.6 \%(1340)$ & $31.0 \%(13)$ & 0.020 \\
Facial injury (AIS $\geq 2)$ & $11.9 \%(965)$ & $42.9 \%(18)$ & $\leq 0.001$ \\
Cervical spine (AIS $\geq 3$ ) & $2.9 \%(232)$ & $31.0 \%(13)$ & $\leq 0.001$ \\
Chest injury (AIS $\geq 2)$ & $42.1 \%(3405)$ & $78.6 \%(33)$ & $\leq 0.001$ \\
Spinal injury (AIS $\geq 2)$ & $19.1 \%(1544)$ & $47.6 \%(20)$ & 30.001 \\
Upper extremity injury (AIS $\geq 2)$ & $27.1 \%(2191)$ & $38.1 \%(16)$ & 0.12 \\
Abdominal injury (AIS $\geq 2)$ & $24.1 \%(1949)$ & $28.6 \%(12)$ & 0.47 \\
Lower extremity injury (AIS $\geq 2)$ & $34.7 \%(2804)$ & $26.2 \%(11)$ & 0.33 \\
\hline
\end{tabular}


Table 3 Interventions and outcome parameters

\begin{tabular}{|c|c|c|c|}
\hline & Non-BCVI & BCVI & $p$ \\
\hline Intubation at scene, $\%(n)^{\mathrm{a}}$ & $38.8 \%(2711)$ & $83.3 \%(30)$ & $<0.001$ \\
\hline Immediate head/neck CT & $85.3 \%(6100)$ & $94.4 \%(34)$ & 0.16 \\
\hline Immediate whole-body CT & $64.6 \%(4581)$ & $86.1 \%(31)$ & 0.008 \\
\hline Any blood transfusion & $10.6 \%(754)$ & $45.7 \%(16)$ & $<0.001$ \\
\hline Ventilator days, mean (SD) median $\beta$ & $3(7) 0$ & $15(22) 10$ & $<0.001$ \\
\hline ICU stay, mean (SD) median $\beta$ & $6(9) 3$ & $20(22) 14$ & $<0.001$ \\
\hline Length of stay, mean (SD) median & $17(17) 12$ & $30(24) 25$ & 0.19 \\
\hline Multiple organ failure, $\%(n) \beta$ & $17.4 \%(830)$ & $72.0 \%(18)$ & $<0.001$ \\
\hline Thromboembolic event, $\%(n) \beta$ & $1.0 \%(43)$ & $8.3 \%(2)$ & 0.026 \\
\hline Mortality within $24 \mathrm{~h}$ after admission & $4.7 \%(384)$ & $26.2 \%(11)$ & $<0.001$ \\
\hline In-hospital mortality & $7.7 \%(619)$ & $38.1 \%(16)$ & $<0.001$ \\
\hline
\end{tabular}

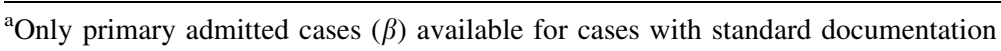

Table 4 Logistic regression analysis: independent predictors for pediatric BCVI

\begin{tabular}{|c|c|c|c|}
\hline Risk factors for BCVI & $\begin{array}{l}\text { Odds ratio } \\
(\mathrm{OR})\end{array}$ & $\begin{array}{l}95 \% \text { confidence } \\
\text { interval }(\mathrm{CI})\end{array}$ & $p$ value \\
\hline $\begin{array}{l}\text { Cervical spine injury } \\
\quad(\text { AIS }>3)\end{array}$ & 8.2 & $3.3-20.3$ & $<0.001$ \\
\hline $\begin{array}{l}\text { Facial injury } \\
(\text { AIS }>2)\end{array}$ & 4.4 & $2.13-9.20$ & $<0.001$ \\
\hline $\begin{array}{l}\text { Basilar skull fracture } \\
\text { (BSF) }\end{array}$ & 2.4 & $1.04-5.45$ & 0.039 \\
\hline $\begin{array}{l}\text { Injury Severity Score } \\
\text { (per point) }\end{array}$ & 1.05 & $1.03-1.07$ & $<0.001$ \\
\hline Age ( $>12$ years $)$ & 1.8 & $0.81-4.05$ & 0.147 \\
\hline Head injury (AIS > 3) & 1.5 & $0.54-4.05$ & 0.455 \\
\hline Road traffic accident & 0.86 & $0.42-1.8$ & 0.692 \\
\hline
\end{tabular}

screening, as both died soon after emergency room admission. Further interventions and outcome parameters are presented in Table 3.

There was an excessive risk for thromboembolic complications in children with BCVI (8.3\% vs. $1 \% ; p=0.026)$, MOF $(72.0 \%$ vs. $17.4 \% ; p \leq 0.001)$ and in-hospital mortality $(7.7 \%$ vs. $38.1 \%, p \leq 0.001)$.

The majority of children with BCVI were admitted to a Level 1 trauma center $(81 \%)$. Primary admission was documented for $85.7 \%$, while $14.3 \%$ were transferred secondarily to a Level 1 trauma center.

\section{Predictors for BCVI}

In a logistic regression analysis model (Table 4), we identified the following variables associated with an increased risk of BCVI in pediatric patients: (1) cervical spine injury (OR 8.2, 95\% CI: 3.3-20.3p $\leq 0.001$ ); (2) facial injury (OR 4.4, 95\% CI 2.2-9.2, $p \leq 0.001$ ); 3 )

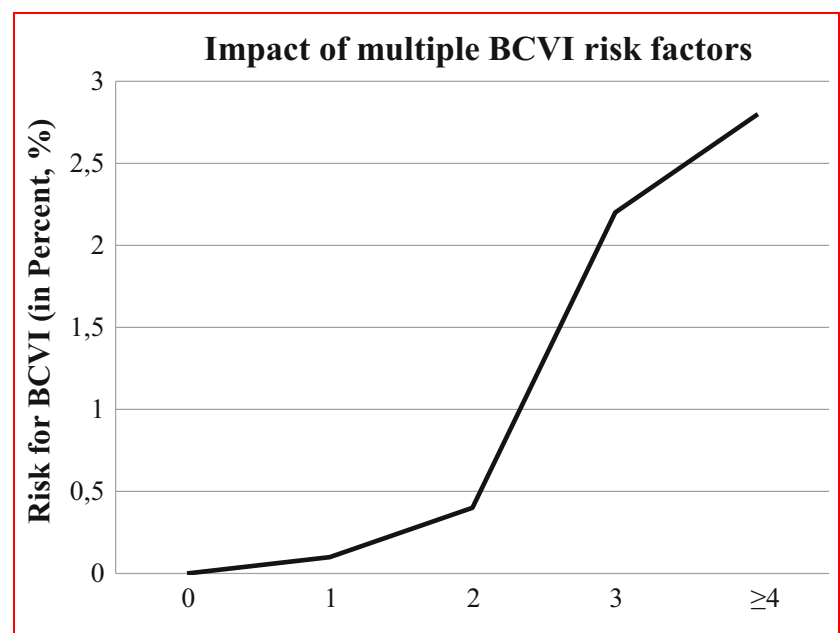

Fig. 5 Cumulative impact of multiple BCVI risk factors

basilar skull fracture (OR 2.4, 95\% CI 1.0-5.5, $p=0.039$ ); and 4) ISS (OR 1.05 [per point], 95\% CI 1.03-1.07, $p$ $\leq 0.001$ ). Road traffic accidents, major head injury (AIS $\geq 3$ points), and age ( $>12$ years) were not significantly associated with BCVI, but the insignificance of age (OR 1.8) may be related to the small number of young children.

We analyzed the cumulative risk for BCVI based on the number of five proposed risk factors (cervical spine injury, facial injury, BSF, age $\geq 12$ years, GCS $\leq 8$ points).

All 42 BCVI patients had at least on risk factor (Fig. 5). There were two patients with one risk factor (BCVI risk $0.1 \%$ ), 12 patients with two risk factors (BCVI risk $0.4 \%$ ), 22 patients with three risk factors (BCVI risk 2.2\%), five patients with four risk factors (BCVI risk 2.8\%), and one patient with five risk factors (BCVI risk 25\%). 


\section{Discussion}

BCVIs are a potentially devastating injury pattern in both adult and pediatric trauma patients. Based on the expanding knowledge about adult BCVI, recent studies have suggested concise screening and management strategies for adult trauma patients $[2,24,25]$. For pediatric patients, these protocols have not yet been established. In this multiinstitutional study, we included a total of 8128 pediatric trauma patients and identified 42 patients with BCVI. This data thereby represents the largest cohort of pediatric BCVI patients outside of North America. The main findings of the current study can be summarized as follows:

1. The overall BCVI incidence is $0.5 \%$ for children between 0 and 17 years.

2. No association with a specific age group was found.

3. The incidence of BCVI is associated with higher injury severity and high-energy trauma. Cervical spine injuries, facial injuries, and basilar skull fractures are predictive factors for the presence of BCVI.

4. CT of the head/neck or whole-body CT scan is part of the diagnostic setup in the vast majority of the pediatric trauma population.

\section{BCVI incidence}

In the only trial with a comparable number of included patients presented in this study, Azarakhsk et al. [26] reported an equal overall incidence of $0.4 \%$ in their multiinstitutional ATOMAC study, based on 23 BCVI cases ( $<15$ years). In contrast, Rossidis et al. [16] previously found a far lower incidence rate of $0.095 \%$, based on 11 patients (age: 0-18 years) who underwent angiographic evaluation, whereas Kopelman et al. [9] observed a higher overall incidence of $0.9 \%$, based on 11 patients between 0 and 14 . These variations of overall incidences might be related to different factors. First, many of the previously conducted studies included only a very limited number of patients. Second, the proportion of children undergoing BCVI screening seems to be different in many trauma centers, which might result in missed injuries under certain conditions.

\section{Carotid and vertebral artery injuries}

In more detail, we found that CAIs $(71.4 \%)$ were more common than VAIs (28.6\%). This finding is in line with Dewan et al., who also reported a higher rate for CAI (82\%) when compared to VAIs (18\%) in children between 0 and 18 years of age. It is possible that the higher prevalence of carotid injuries is caused by a higher vulnerability related to the anatomic exposure. In our study, we did not observe a special predisposition for BCVI in a specific age group. However, high-energy mechanisms were more often observed in children sustaining BCVI (70.4\% vs. $85.4 \%, p=0.039)$. In accordance, overall injury severity was also significantly higher in these patients. These associations might explain the increased incidence of BCVI in adults compared to the pediatric population since adults are more often involved in highenergy trauma, such as severe road traffic accidents.

\section{Imaging}

The vast majority of patients included in this study underwent head/neck (94.4\%) or whole-body CT $(86.1 \%)$, independent of the presence of BCVI. Therefore, no specific reticence or restricted use for CT scans was observed in the current pediatric study population. In fact, we observed very comparable rates for CT of the head/neck region $(92.5 \%)$ or whole-body CT $(87.1 \%)$ in a recently published adult BCVI patient series [1]. Only two children were identified who did not undergo CT-based screening, as both died early in the emergency room phase. We consider the high proportion of imaged patients as strength of the study since the under-recognition of BCVI is a wellanticipated phenomenon. However, we are unable to comment on diagnostic modalities other than CT, since conventional angiography, MRA, Doppler, and delayed CTAs are not captured within the trauma registry.

While the CTA is currently the procedure of choice for severely injured patients and pediatric BCVI screening in many centers, no evidence-based standard has been implemented yet. However, in order to reduce missed BCVI and to limit the radiation exposure particularly in our very young patients at the same time, our data may help clinicians in weighting up radiation exposure and the expected risk for BCVI based on the number of risk factors.

\section{BCVI risk factors}

Rossidis et al. [16] already suggested that each pediatric BCVI patients sustained at least one risk factor. In our series, the risk increased from $0 \%$ (no risk factor) up to $25 \%$ in five risk factors (Fig. 5). The presence of cervical, basilar skull, and facial fractures represent predictive factors for BCVI, whereas severe TBI per se is not an independent risk factor. Age was not a significant risk factor, but this might be related to the small number of very young children (OR 1.8). We found the most evident differences in cervical spine injuries ( $31 \%$ vs. $2.9 \%, p \leq 0.001$ ). Kopelman et al. [9] reported that $17 \%$ of pediatric BCVI patients suffered from a basilar skull fracture (BSF); at $31 \%$, we observed an even higher incidence. The number 
of children with a loss of consciousness (LOC; 71.4\%) in our study was comparable to a study by Ravindra et al., who described a similar risk for LOC (72.2\%) [27].

While some studies provide lower or no mean ISS values, ranging from 22.4 [26] to 26.7 points [16], the mean ISS in our series is one of the highest ever published in the context of pediatric BCVI. Despite this high ISS, the incidence of BCVI was significantly lower compared to studies that investigated adult trauma patients [1,2]. This might suggest that children are potentially less susceptible to cerebrovascular injuries when compared to adults and that the proposed theoretical risk factors (e.g., head-body ratio, weak neck musculature) do not actually result in increased BCVI rates in children. The high injury severity in our study might be related to the nature of the trauma registry, which was specifically designed to capture major trauma victims. In this context, we also feel the observed differences in injury severity and the rate of head injuries have contributed to higher mortality $(38.1 \%)$ in our data when compared to studies that reported a lower range, between 13.9 and $27 \%[9,16]$.

\section{Level of care}

Dewan et al. [28] analyzed whether various trauma centers (four academic pediatric trauma centers) correlated with the rate of mortality, and found no impact. In the current study, we observed that the majority of pediatric BCVI patients were managed in Level 1 centers, but $19 \%$ were admitted to Level 2 trauma centers. In pediatric trauma patients without BCVI, more patients were managed outside Level 1 centers $(24.7 \%, n=1999)$. This can be explained by the higher overall injury severity of patients with BCVI, which might be associated with an increased frequency of admission to a Level I trauma center. However, in Germany, no coverage by dedicated pediatric trauma centers has been established, so the comparison of these findings may be limited.

\section{Limitations and strengths}

We acknowledge further specific limitations associated with our study. First, the TraumaRegister $\mathrm{DGU}^{\circledR}$ cannot give detailed insights into the onset and course of neurologic symptoms, other than pupil reaction and GCS values. Furthermore, confounders of the neurologic outcome, including severe head and spinal cord injuries, could not be excluded due to a severely injured patient cohort. Another limiting factor was the use of AIS values to differentiate the severity of BCVI, instead of the Biffl system. Currently, the Biffl grading system is widely appreciated [4, 29-33], but participating trauma centers in Germany, Switzerland, and Austria are more familiar with the uniform AIS classification since it is the single most popular system used throughout the study period within the trauma registry. Furthermore, participating trauma centers use different CT scanners and have established various CT protocols. Therefore, we are unable to comment on center-specific diagnostic accuracy.

However, we feel that the high number of evaluated pediatric patients, consistent injury scoring, and the high rate of CT-based screening due to multiple injuries in children as a strength of the study allow us to draw the following conclusion.

\section{Conclusion}

Pediatric BCVI is less frequent when compared to adult trauma patients, despite an aggressive utilization of CT imaging techniques. In this context, CAI is observed more often compared to VAI. The risk factors for BCVI in children seem to mimic those reported for adult trauma patients and include cervical spine and facial injuries, basilar skull fractures, and general injury severity. Pediatric BCVI is associated with a significant burden of complications and excessive mortality; therefore, further studies are required to improve clinical pathways.

Acknowledgements The authors would like to thank the internal review board of the TraumaRegister $\mathrm{DGU}^{\circledR}$ for their substantial contribution. Furthermore, we thank all participating trauma centers (complete list at www.traumaregister.de).

\section{Compliance with ethical standards}

Conflict of interest The authors declare that they have no conflict of interest.

\section{References}

1. Weber CD, Lefering R, Kobbe P, Horst K, Pishnamaz M, Sellei RM, Hildebrand F, Pape HC (2017) Blunt cerebrovascular artery injury and stroke in severely injured patients: an international multicenter analysis. World J Surg. https://doi.org/10.1007/ s00268-017-4408-6

2. Bromberg WJ, Collier BC, Diebel LN, Dwyer KM, Holevar MR, Jacobs DG, Kurek SJ, Schreiber MA, Shapiro ML, Vogel TR (2010) Blunt cerebrovascular injury practice management guidelines: the Eastern Association for the Surgery of Trauma. J Trauma 68:471-477. https://doi.org/10.1097/TA. 0b013e3181cb43da

3. Berne JD, Norwood SH, McAuley CE, Vallina VL, Creath RG, McLarty J (2001) The high morbidity of blunt cerebrovascular injury in an unscreened population: more evidence of the need for mandatory screening protocols. J Am Coll Surg 192:314-321

4. Biffl WL, Moore EE, Elliott JP, Ray C, Offner PJ, Franciose RJ, Brega KE, Burch JM (2000) The devastating potential of blunt vertebral arterial injuries. Ann Surg 231:672-681 
5. McNutt MK, Kale AC, Kitagawa RS, Turkmani AH, Fields DW, Baraniuk S, Gill BS, Cotton BA, Moore LJ, Wade CE, Day A, Holcomb JB (2017) Management of blunt cerebrovascular injury (BCVI) in the multisystem injury patient with contraindications to immediate anti-thrombotic therapy. Injury. https://doi.org/10. 1016/j.injury.2017.07.036

6. Shafafy R, Suresh S, Afolayan JO, Vaccaro AR, Panchmatia JR (2017) Blunt vertebral vascular injury in trauma patients: ATLS(R) recommendations and review of current evidence. J Spine Surg 3:217-225. https://doi.org/10.21037/jss.2017.05.10

7. Shahan CP, Magnotti LJ, McBeth PB, Weinberg JA, Croce MA, Fabian TC (2016) Early antithrombotic therapy is safe and effective in patients with blunt cerebrovascular injury and solid organ injury or traumatic brain injury. J Trauma Acute Care Surg 81:173-177. https://doi.org/10.1097/ta.0000000000001058

8. White D, Place R, Michael T, Hoffman E, Gordon PM, Visich P (2017) The relationship between coronary artery disease risk factors and carotid intima-media thickness in children. J Pediatr 190:38-42. https://doi.org/10.1016/j.jpeds.2017.07.034

9. Kopelman TR, Berardoni NE, O'Neill PJ, Hedayati P, Vail SJ, Pieri PG, Feiz-Erfan I, Pressman MA (2011) Risk factors for blunt cerebrovascular injury in children: do they mimic those seen in adults? J Trauma 71:559-564. https://doi.org/10.1097/ta. 0b013e318226eadd discussion 564

10. Golden J, Isani M, Bowling J, Zagory J, Goodhue CJ, Burke RV, Upperman JS, Gayer CP (2016) Limiting chest computed tomography in the evaluation of pediatric thoracic trauma. J Trauma Acute Care Surg 81:271-277. https://doi.org/10.1097/ ta.0000000000001110

11. Holscher CM, Faulk LW, Moore EE, Cothren Burlew C, Moore HB, Stewart CL, Pieracci FM, Barnett CC, Bensard DD (2013) Chest computed tomography imaging for blunt pediatric trauma: not worth the radiation risk. J Surg Res 184:352-357. https://doi. org/10.1016/j.jss.2013.04.044

12. Miele V, Di Giampietro I, Ianniello S, Pinto F, Trinci M (2015) Diagnostic imaging in pediatric polytrauma management. Radiol Med 120:33-49. https://doi.org/10.1007/s11547-014-0469-x

13. Bayer J, Reising K, Kuminack K, Sudkamp NP, Strohm PC (2015) Is whole-body computed tomography the standard workup for severely-injured children? Results of a survey among German Trauma Centers. Acta Chir Orthop Traumatol Cechoslov 82:332-336

14. Jindal A, Velmahos GC, Rofougaran R (2002) Computed tomography for evaluation of mild to moderate pediatric trauma: are we overusing it? World J Surg 26:13-16. https://doi.org/10. 1007/s00268-001-0174-5

15. Kim PK, Zhu X, Houseknecht E, Nickolaus D, Mahboubi S, Nance ML (2005) Effective radiation dose from radiologic studies in pediatric trauma patients. World J Surg 29:1557-1562. https://doi.org/10.1007/s00268-005-0106-x

16. Rossidis AC, Tharakan SJ, Bose SK, Shekdar KV, Nance ML, Blinman TA (2017) Predictors of pediatric blunt cerebrovascular injury. J Pediatr Surg. https://doi.org/10.1016/j.jpedsurg.2017.10. 037

17. Desai NK, Kang J, Chokshi FH (2014) Screening CT angiography for pediatric blunt cerebrovascular injury with emphasis on the cervical "seatbelt sign". AJNR Am J Neuroradiol 35:1836-1840. https://doi.org/10.3174/ajnr.A3916

18. Dgu T (2014) 20 years TraumaRegister DGU((R)): development, aims and structure. Injury 45(Suppl 3):S6-s13. https://doi.org/10. 1016/j.injury.2014.08.011

19. Baker SP, O'Neill B, Haddon W Jr, Long WB (1974) The injury severity score: a method for describing patients with multiple injuries and evaluating emergency care. J Trauma 14:187-196

20. Greenspan L, McLellan BA, Greig H (1985) Abbreviated injury scale and injury severity score: a scoring chart. J Trauma 25:60-64

21. Aho K, Harmsen P, Hatano S, Marquardsen J, Smirnov VE, Strasser T (1980) Cerebrovascular disease in the community: results of a WHO collaborative study. Bull World Health Organ 58:113-130

22. Vincent JL, de Mendonca A, Cantraine F, Moreno R, Takala J, Suter PM, Sprung CL, Colardyn F, Blecher S (1998) Use of the SOFA score to assess the incidence of organ dysfunction/failure in intensive care units: results of a multicenter, prospective study. Working group on "sepsis-related problems" of the European Society of Intensive Care Medicine. Crit Care Med 26:1793-1800

23. Bone RC, Balk RA, Cerra FB, Dellinger RP, Fein AM, Knaus WA, Schein RM, Sibbald WJ (1992) Definitions for sepsis and organ failure and guidelines for the use of innovative therapies in sepsis. The ACCP/SCCM Consensus Conference Committee. American College of Chest Physicians/Society of Critical Care Medicine. Chest 101:1644-1655

24. Bruns BR, Tesoriero R, Kufera J, Sliker C, Laser A, Scalea TM, Stein DM (2014) Blunt cerebrovascular injury screening guidelines: what are we willing to miss? J Trauma Acute Care Surg 76:691-695. https://doi.org/10.1097/TA.0b013e3182ab1b4d

25. Harrigan MR, Marques MB, Williams LA 3rd (2017) A safe and effective management strategy for BCVI. J Trauma Acute Care Surg 82:228. https://doi.org/10.1097/ta.0000000000001288

26. Azarakhsh N, Grimes S, Notrica DM, Raines A, Garcia NM, Tuggle DW, Maxson RT, Alder AC, Recicar J, Garcia-Filion P, Greenwell C, Lawson KA, Wan JY, Eubanks JW 3rd (2013) Blunt cerebrovascular injury in children: underreported or underrecognized? A multicenter ATOMAC study. J Trauma Acute Care Surg 75:1006-1011. https://doi.org/10.1097/ta. 0b013e $31829 \mathrm{~d} 3526$ discussion 1011-1002

27. Ravindra VM, Riva-Cambrin J, Sivakumar W, Metzger RR, Bollo RJ (2015) Risk factors for traumatic blunt cerebrovascular injury diagnosed by computed tomography angiography in the pediatric population: a retrospective cohort study. J Neurosurg Pediatr 15:599-606. https://doi.org/10.3171/2014.11.peds14397

28. Dewan MC, Ravindra VM, Gannon S, Prather CT, Yang GL, Jordan LC, Limbrick D, Jea A, Riva-Cambrin J, Naftel RP (2016) Treatment practices and outcomes after blunt cerebrovascular injury in children. Neurosurgery 79:872-878. https://doi.org/10. 1227/neu.0000000000001352

29. Biffl WL, Moore EE, Offner PJ, Brega KE, Franciose RJ, Elliott JP, Burch JM (1999) Optimizing screening for blunt cerebrovascular injuries. Am J Surg 178:517-522

30. Biffl WL, Moore EE, Ryu RK, Offner PJ, Novak Z, Coldwell DM, Franciose RJ, Burch JM (1998) The unrecognized epidemic of blunt carotid arterial injuries: early diagnosis improves neurologic outcome. Ann Surg 228:462-470

31. Scott WW, Sharp S, Figueroa SA, Eastman AL, Hatchette CV, Madden CJ, Rickert KL (2015) Clinical and radiographic outcomes following traumatic grade 3 and 4 carotid artery injuries: a 10-year retrospective analysis from a level 1 trauma center. The Parkland carotid and vertebral artery injury survey. J Neurosurg 122:610-615. https://doi.org/10.3171/2014.10.jns14875

32. Scott WW, Sharp S, Figueroa SA, Eastman AL, Hatchette CV, Madden CJ, Rickert KL (2015) Clinical and radiographic outcomes following traumatic grade 1 and 2 carotid artery injuries: a 10-year retrospective analysis from a level I trauma center. The 
Parkland carotid and vertebral artery injury survey. J Neurosurg. https://doi.org/10.3171/2015.1.jns14642

33. Sliker CW, Shanmuganathan K, Mirvis SE (2008) Diagnosis of blunt cerebrovascular injuries with 16-MDCT: accuracy of whole-body MDCT compared with neck MDCT angiography.
AJR Am J Roentgenol 190:790-799. https://doi.org/10.2214/ajr. 07.2378

Publisher's Note Springer Nature remains neutral with regard to jurisdictional claims in published maps and institutional affiliations. 